\title{
Mammographycally occult high grade ductal carcinoma in situ (DCIS) as second primary breast cancer, detected with MRI: a case report
}

\author{
Marta Zebic-Sinkovec ${ }^{1}$, Maksimiljan Kadivec ${ }^{1}$, Gasper Podobnik ${ }^{1}$, Erik Skof ${ }^{2}$, Marko Snoj ${ }^{3}$ \\ ${ }^{1}$ Department of Radiology, Institute of Oncology Ljubljana, Slovenia \\ ${ }^{2}$ Department of Medical Oncology, Institute of Oncology Ljubljana, Slovenia \\ ${ }^{3}$ Department of Surgery, Institute of Oncology Ljubljana, Slovenia
}

Received 17 March 2010

Accepted 27 May 2010

Correspondence to: Marta Zebič-Šinkovec, MD, Department of Radiology, Institute of Oncology Ljubljana,

Zaloška 2, SI-1000 Ljubljana, Slovenia. Phone: +386 15879 940; Fax: +386 15879 407; E-mail: MZebic@onko-i.si

Disclosure: No potential conflicts of interest were disclosed.

Background. Contralateral breast cancer $(C L B)$ is the most common second primary breast cancer in patients diagnosed with breast cancer. The majority of patients harbouring CLB tumours develop the invasive disease. Almost all invasive carcinomas are believed to begin as ductal carcinoma in situ (DCIS) lesions. The sensitivity of MRI for DCIS is much higher than that of mammography.

Case report. We report the case of a woman who was treated with breast conserving therapy 10 years ago. At that time the invasive medullary carcinoma was diagnosed in the left breast. Ten years later mammographically occult DCIS was diagnosed with MRI-guided core biopsy in contralateral breast.

Conclusions. There might be a potential role of MRI screening as part of an annual follow-up for patients diagnosed with breast cancer.

Key words: high-grade DCIS; second primary breast cancer; MRI

\section{Introduction}

Contralateral breast cancer (CLB) is the most common second primary breast cancer in patients diagnosed with breast cancer. ${ }^{1}$ The annual risk of developing any CLB remains constant at approximately $0.75 \%$ and persists for at least 20 years after the treatment. The majority of patients $(83 \%)$ harbouring CLB tumours develop the invasive disease. ${ }^{2}$ There is little data on the use of MRI as a screening tool to detect a recurrence after the breast-conserving therapy. Gorechland et al. concluded that MRI screening would not have been cost-effective and was unlikely to have improved the overall survival. ${ }^{3}$ However, the role of MRI in detection of invasive carcinoma had already been known, Kuhl et al. published in 2007 that MRI is more sensitive for detecting ductal carcinoma in situ (DCIS) than mammography ( $92 \%$ vs. $56 \%$ ), especially for high-grade
DCIS without necrosis (92\% vs. $35 \%) .{ }^{4}$ Almost all invasive carcinomas are believed to begin as DCIS lesions. ${ }^{5}$ Therefore, some invasive carcinomas can be prevented by a timely intervention on the basis of MRI findings.

\section{Case report}

A 47-year-old female patient was treated by breast conserving surgery in 1999. At that time invasive medullary carcinoma was diagnosed in her left breast. The dissection of axilla has been done and there was no metastatic lymph node. She received adjuvant chemotherapy and a radiation therapy. She had the regular clinical and mammographic follow-up. In April 2009 her last mammography was obtained (Figure 1). Radiological findings were evaluated according to the Breast Imaging 

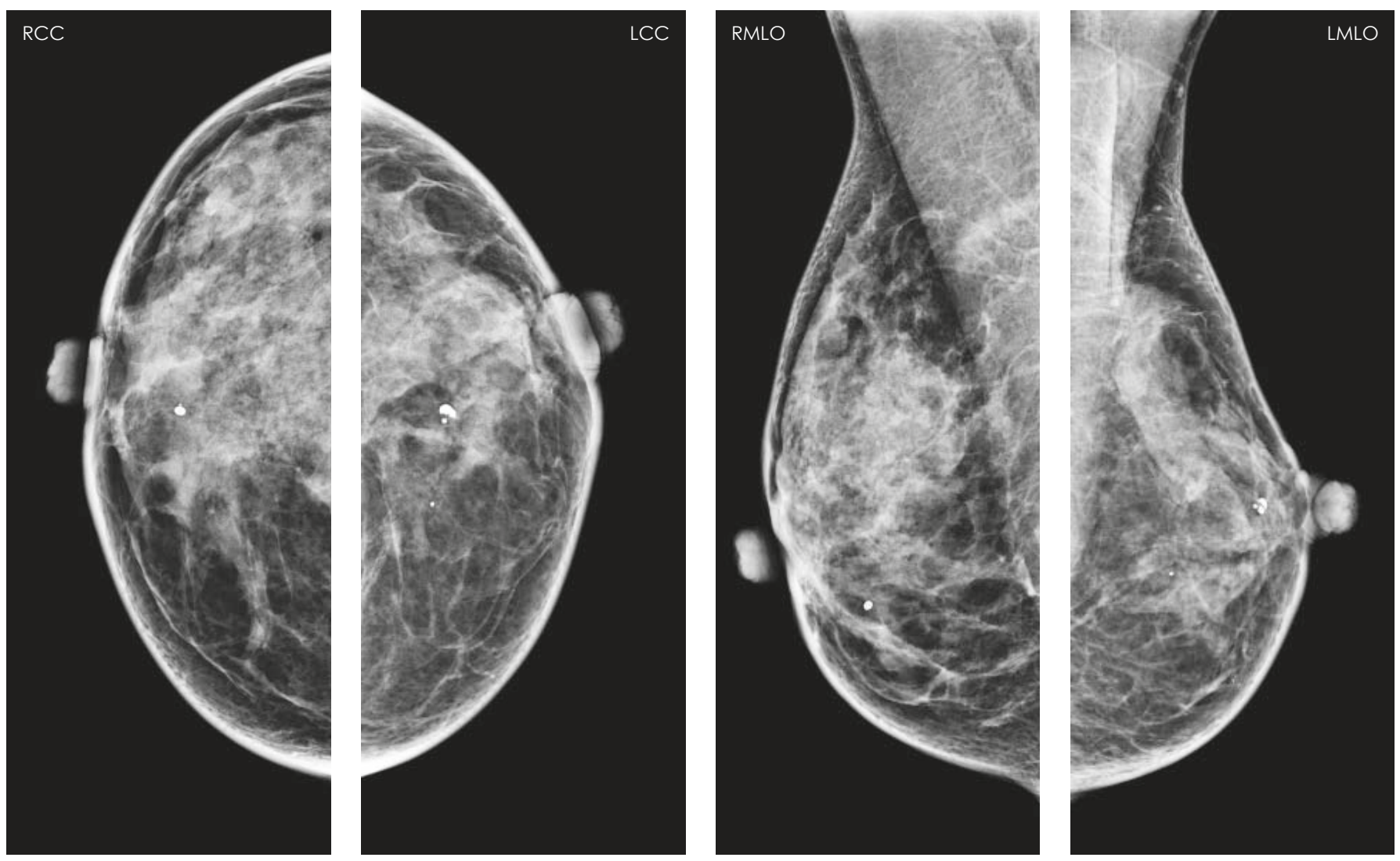

FIGURE 1. The mammograms were categorized as BI-RADS 2 (cyst, benign calcifications, postoperative changes). The breast density was categorized as ACR III. There was no change in comparison with previous mammograms.

Reporting and Data System by American College of Radiology (Figure 1).

In November 2009, she visited her doctor earlier because of changes in her right nipple. The nipple became retracted. She also had pain in her breast. Breast MRI was performed, using a 1.5-T magnet with a dedicated bilateral breast surface coil with prone position. The imaging protocol and parameters were as follows: axial T1-weighted image and short-tau inversion recovery (STIR) of both breasts were obtained with $3 \mathrm{~mm}$ slice thickness. Next, T1-weighted images were acquired using a 3D fast low angle shot (FLASH) through both breasts. Pre-contrast images were obtained in the axial plane with a slice thickness of $1.0 \mathrm{~mm}$ with a distance factor $20 \%$ before the administration of the contrast agent. Then, five sequential contrastenhanced images were acquired at every $1 \mathrm{~min}$ $23 \mathrm{~s}$. The MRI findings were categorized according to Breast Imaging Reporting and Data system (BIRADS) lexicon.

After a gadolinium injection and subtraction a bilateral enhancement was seen: On the left side there was a $7 \times 5 \mathrm{~mm}$ mass-like enhancement in the scar area. The margins were round and well circumscribed, the enhancement was homogeneous, and kinetic was $173 \%$ initial enhancement with plato BI-RADS 2 (Figure 2).

On the right there was a non-masslike enhancement. The enhancement pattern was ductal-linear in distribution measured $8 \times 3 \mathrm{~mm}$. The internal enhancement was homogenous -BI-RADS 3-4 (Figure 3).

On the precontrast T2-weighted sequence there was a hiperintensive signal in the area of ductal enhancement in the right breast. There were small cysts bilaterally (Figure 4).

The targeted ultrasound was performed, using 5-12 MHz linear transducer (Toshiba Aplio, Nasu, Japan). In the right breast there was no pathology. In the left breast there was a small tumour $5 \times 4$ $\mathrm{mm}$ categorized as BI-RADS 4 (Figure 5). The fine needle US guided biopsy was performed and cytology was inconclusive. During the procedure the patient was very anxious and difficult to communicate with.

Because of the MRI finding in the right breast (mammogaphicaly occult, targeted ultrasound negative) and because of the patient's history the 


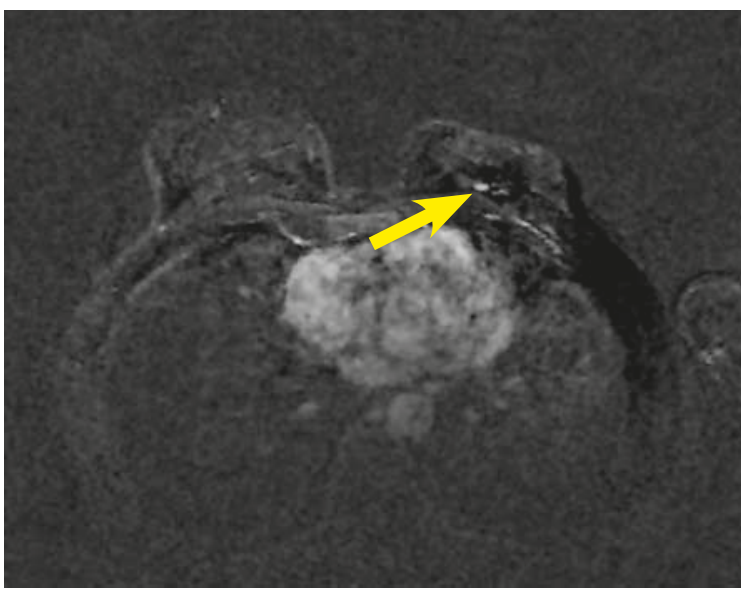

FIGURE 2. Axial T1-weighted image after Gadolinium injection ( $2^{\text {nd }}$ minute) and subtraction, focal enhancement $7 \times 5 \mathrm{~mm}$ in the left breast in the prepectoral region (arrowhead).

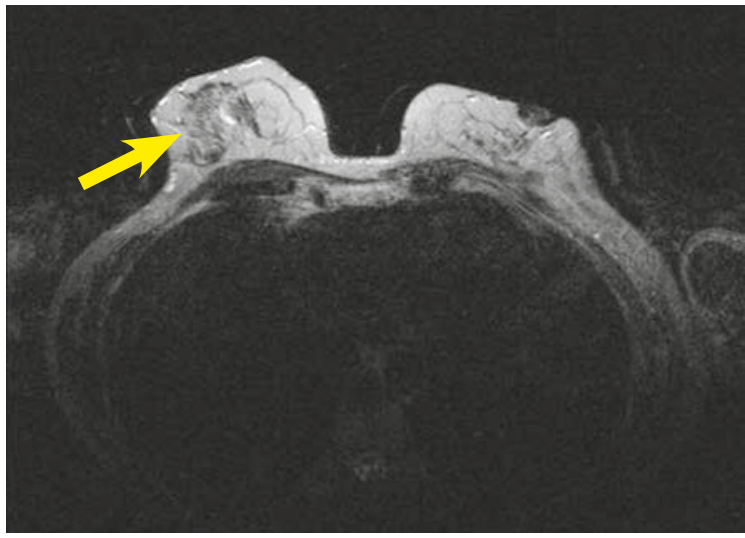

FIGURE 4. Axial T2-weighted image, a hiperintensive signal in the right breast (arrowhead).

MRI-guided core biopsy was performed. MRIguided vacuum-assisted breast biopsy was performed with MRI-supported Breast Immobilization and Biopsy System with the 4-channel breast coil in prone position. Axial T1- weighted images were acquired using a 3D FLASH through both breasts. Precontrast images were obtained in the axial plane with a slice thickness of $1.0 \mathrm{~mm}$ with distance factor $20 \%$. Twenty seconds after contrast agent had been injected, another axial T1-3D FLASH sequence was performed with an injection of $0.1 \mathrm{mmol} / \mathrm{kg}$ of body weight of gadopentetate dimeglumine. Biopsy was performed with a 9-gauge MRI compatible vacuum-assisted biopsy. The biopsy site was marked with a titanium clip. "Postclip" axial 3D FLASH was performed to assess clip deployment.

The histological finding was DCIS-high grade, without any calcification. The patient was operated. The breast conserving therapy was performed.

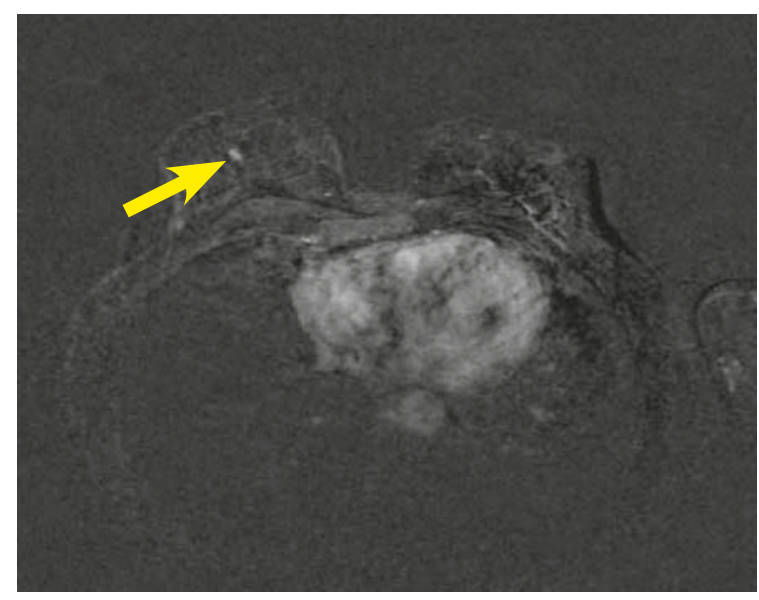

FIGURE 3. Axial T1-weighted image after Gadolinium injection ( $2^{\text {nd }}$ minute) and subtraction, ductal homogenous enhancement in the right breast $8 \times 3 \mathrm{~mm}$ (arrowhead).

The clip in the right breast was localized by the radioguided occult lesion localization (ROLL) method under X-ray guidance. The lesion in her left breast was localized by ROLL method under US guidance. The pathologic results were the remnant foci of high-grade DCIS in the right breast and benign changes in the left breast.

\section{Discussion}

The screening MRI has not yet been included in surveillance for patients treated by a breast-conserving therapy. However, the patient visited her doctor earlier because of changes in her right nipple, what demonstrated the importance of the breastself examination. ${ }^{6}$ In addition, in our case MRI was performed because the patient had retracted nipple and dense breast. ${ }^{3}$ DCIS was represented as a ductal-linear homogenous enhancement on MRI images. The ductal-linear homogenous enhancement is a type of non-masslike enhancement. ${ }^{78}$ The path of enhancement follows the galactophoric system. The internal feature of the enhancement was homogenous in our case. DCIS and inflammatory disease are the most common causes for such a type of enhancement. The targeted ultrasound was negative, as we had expected.

Among the non-masslike enhancement detected initially on MRI, only $11 \%$ could be retrospectively detected by ultrasound and sonographically occult lesions have $22 \%$ probability of malignancy. ${ }^{9-12}$ Although the ductal enhancement was small, it measured only $8 \times 3 \mathrm{~mm}$, we decided to perform MRI-guided core biopsy and the histological result was conclusive. ${ }^{13,14}$ There was also a lesion which 
was incidentally found in the scar area of the left breast, which finally proved to be benign.

High-grade DCIS with no calcifications is not easy to diagnose by mammography due to the lack of typical malignant calcifications or masses, especially in dense breasts. Calcifications with or without mass are more common in women under 50 years..$^{11}$ Autopsy studies have shown that almost $9 \%$ of women have undetected DCIS. ${ }^{15}$

Almost all invasive carcinomas are believed to begin as DCIS lesions but the time course of transition is unknown. Whether all DCIS will ultimately evolve to the invasive disease is unclear. ${ }^{16,17}$ In 2007 an article was published, that sensitivity of MRI for high-grade DCIS is much higher than that of mammography (92\% vs. $56 \%$ ), especially for high grade DCIS without necrosis (97\% vs. $35 \%) .{ }^{4}$ If we pick up all cases of DCIS we would prevent virtually all cases of breast cancer, including CLB. CLB is the most common second primary breast cancer in patients diagnosed with breast cancer. The annual risk of developing any CLB remains constant at $0.75 \%$ per year after the treatment and persists for at least 20 years. The majority of patients (83\%) harbouring CLB tumours develop invasive disease. ${ }^{2}$ The detection of second breast cancers in the asymptomatic phase leads to the detection of early-stage cancer and it improves the relative survival alike in other cancer's localisations between $27 \%$ to $47 \% .^{18,19}$

In conclusion, by the Breast MRI Guidelines from the European Society of Breast Imaging ${ }^{14}$, currently there is not sufficient evidence to recommend the screening with MRI to patients treated by breast conserving surgery. But we might say that our case, in accordance to the European Guidelines, justifies MRI as a problem-solving modality when: the findings of conventional imaging are inconclusive and it is impossible to image sufficiently the primary tumour region after the conservative therapy with mammography.

\section{References}

1. Wedam SB, Swain SM. Contralateral breast cancer: Where does it all begin? J Clin Oncol 2005; 23: 4585-7.

2. Hill-Kayser CE, Harris EE, Hwang WT, Solin U. Twenty-year incidence and patterns of contralateral breast cancer after breast conservation treatment with radiation. Int J Radiat Oncol Biol Phys 2006; 66: 1313-9.

3. Gorechlad JW, McCabe EB, Higgins JH, Likosky DS, Lewis PJ, Rosenkranz KM, et al. Screening for recurrences in patients treated with breast-conserving surgery: is there a role for MRI? Ann Surg Oncol 2008; 15: 1703-9.

4. Kuhl CK, Schrading S, Bieling HB, Wardelmann E, Leutner CC, Koenig R, et al. MRI for diagnosis of pure ductal carcinoma in situ: a prospective observational study. Lancet 2007; 370: 485-92.

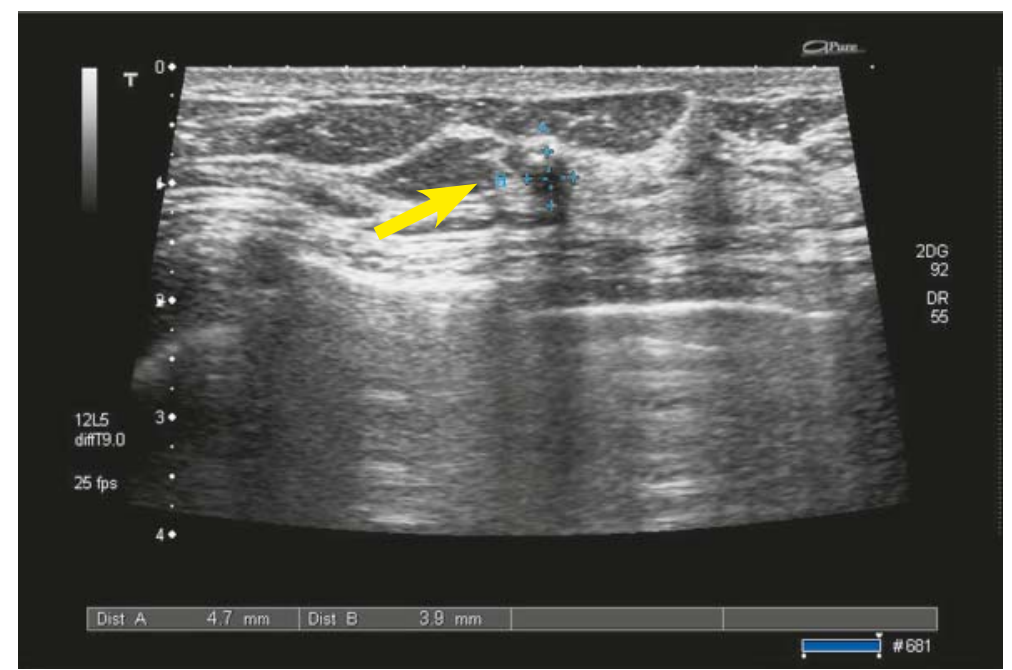

FIGURE 5. Small lesion in the left breast $5 \times 4 \mathrm{~mm}$, transonic with unsharp margins, vertically orientated, BI-RADS 4 (arrowhead).

5. Thomson JZ, Evans AJ, Pinder SE, Burrell HC, Wilson ARM, Ellis IO. Growth pattern of ductal carcinoma in situ (DCIS): a retrospective analysis based on mammographic findings. Brit J Cancer 2001; 85: 225-7.

6. Plesnicar A, Golicnik M, Fazarinc IK, Kralj B, Kovac V, Plesnicar BK. Attitudes of midwifery students towards teaching breast-self examination. Radiol Oncol 2010; 44: 52-6.

7. Thomassin-Naggara I, Salem C, Darai E, Bazot M, Uzan S, Marsault C Chopier J. Non-masslike enhancement on breast MRI: interpretation pearls. J Radiol 2009; 90 (3 Pt 1): 269-75.

8. Sakamoto N, Tozaki M, Higa K, Tsunoda Y, Ogawa T, Abe S, et al. Categorization of non-mass-like breast lesions detected by MRI. Breast Cancer 2008; 15: 241-6.

9. LaTrenta LR. Breast lesions detected with MR imaging: utility and histopathologic importance of identification with US. Radiolology 2003; 227: 856-61.

10. Abe Schmidt RA, Shah RN, Shimauchi A, Kulkarni K, Sennett CA, Newstead GM. MR-directed ("Second-Look") ultrasound examination for breast lesions detected initially on MRI: MR and sonographic findings. AJR Am J Roentgenol 2010; 194: 370-7.

11. Podkrajsek M, Zgajnar J, Hocevar M. What is the most common mammographic appearance of T1a and T1b invasive breast cancer? Radiol Oncol 2008; 42: 173-80

12. Kocijancic I. Diagnostic imaging of small amounts of pleural fluid: Pleural effusion vs. physioloic pleural fluid. Coll Antropol 2007; 31: 1195-99.

13. Kuhl CK, Morakkabati N, Leutner CC, Schmiedel A, Wardelmann E, Schild $\mathrm{HH}$. MR imaging-guided large-core (14-gauge) needle biopsy of small lesions visible at breast imaging lone. Radiology 2001; 220: 31-9.

14. Welch HG, Black WC. Using autopsy series to estimate the disease "reservoir" for ductal carcinoma in situ of the breast: how much more breast cancer can we find? Ann Intern Med 1997; 127: 1023-8.

15. Mann RM, Kuhl CK, Kinkel K, Boetes C. Breast MRI: guidelines from the European Society of Breast Imaging. Eur Radiol 2008; 18: 1307-18.

16. Leonard GD, Swain SM. Ductal carcinoma in situ, complexities and challenges. J Natl Cancer Inst 2004; 96: 906-20.

17. Erbas B, Provenzano E, Arnes J, Gertig D. The natural history of ductal carcinoma in situ of the breast: a rewiev. Breast Cancer Res Treat 2006; 97: 135-44.

18. Debevec $L$, Jeric $T$, Kovac $V$, Bitenc $M$, Sok $M$. Is there any progress in routine management of lung cancer patients? A comparative analysis of an institution in 1996 and 2006. Radiol Oncol 2009; 43: 47-53.

19. Houssami N, Ciatto S, Martinelli F, Bonardi R, Duffy SW. Early detection of second breast cancers improves prognosis in breast cancer survivors. Ann Oncol 2009; 20: 1505-10. 